\title{
Biweekly fotemustine schedule for recurrent glioblastoma in the elderly: activity and toxicity assessment of a multicenter study
}

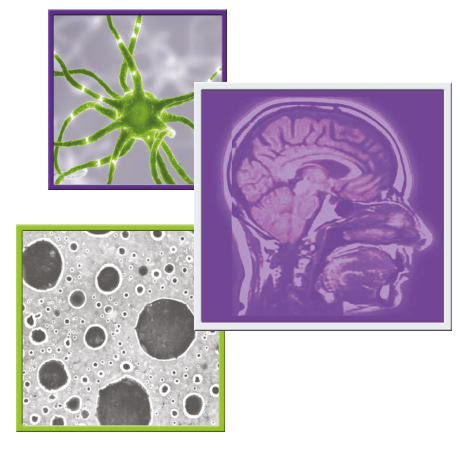

\author{
Raffaele Addeo*,1, Giuseppe Lamberti ${ }^{2}$, Giorgia Simonetti ${ }^{3}$, Patrizia lodice ${ }^{1}$, Alfredo \\ Marinelli ${ }^{4}$, Liliana Montella ${ }^{1}$, Salvatore Cappabianca ${ }^{5}$, Paola Gaviani ${ }^{3}$, Michele Caraglia ${ }^{5}$, \\ Salvatore Del Prete ${ }^{1} \&$ Antonio Silvani ${ }^{3}$ \\ ${ }^{1}$ Medical Oncology Unit, 'San Giovanni di Dio' Hospital, A.S.L. Napoli 2 Nord, Frattamaggiore (Naples), Italy \\ ${ }^{2}$ Department of Experimental, Diagnostic \& Specialty Medicine, S.Orsola-Malpighi University Hospital, Bologna, Italy \\ ${ }^{3}$ Neuro Oncology Unit, Fondazione IRCSS 'Carlo Besta', Milano, Italy \\ ${ }^{4}$ Department of Clinical Medicine \& Surgery, University Federico II of Naples, Naples, Italy \\ ${ }^{5}$ Department of Precision Medicine, University of Campania 'L. Vanvitelli', Naples, Italy \\ *Author for correspondence: raffaeleaddeo19@gmail.com
}

\begin{abstract}
Aim: To assess the efficacy and safety of alternative fotemustine administration schedule in elderly patients with recurrent glioblastoma. Patients $\&$ methods: Patients aged $>65$ years with recurrent glioblastoma received fotemustine $\left(80 \mathrm{mg} / \mathrm{m}^{2}\right.$; days $1,15,30,45$ and 60 , and subsequently every 4 weeks). Primary end point was progression-free survival (PFS) rate at 6 months. Main secondary end point was safety. Results: 58 patients were enrolled at two centers. PFS at 6 months was $47 \%$ (27 patients) and overall response rate was $29 \%$. Median PFS and survival were 6 and 7 months, respectively, and longer in responders versus nonresponders. No grade 3-4 hematological toxicities occurred. Conclusion: The alternative fotemustine administration schedule was an effective and safe treatment for recurrent glioblastoma in elderly patients.
\end{abstract}

First draft submitted: 29 January 2019; Accepted for publication: 8 May 2019; Published online: 10 July 2019

Keywords: alkylating • elderly • fotemustine $\bullet$ glioblastoma $\bullet$ high-grade glioma $\bullet$ nitrosourea • recurrent

Glioblastoma (GBM) is the most common malignant primary brain tumor. Its incidence increases with age, and a worse prognosis is observed in older patients [1]. Among patients with GBM, $>50 \%$ are aged $>65$ years, and the median survival in this population is $<6$ months [2].

In recent years, attention has been focused on elderly GBM patients, defined as those aged $>65$ years [3-6]. Elderly GBM patients usually exhibit a worse outcome and suffer from more adverse events versus younger patients due to comorbidities, lower organ function reserves, less aggressive treatments, increased toxicity of therapies and more aggressive tumor behavior $[4,7,8]$.

The current standard of care for newly diagnosed GBM in elderly patients is a short-course radiotherapy plus concomitant and adjuvant temozolomide (TMZ), which has been proven to prolong median survival versus radiotherapy alone [9]. In patients unsuitable for combined chemo- and radiation therapy, either TMZ alone in patients with O6-methylguanine-DNA-methyltransferase (MGMT) methylation or hypofractionated radiation therapy may be offered [10].

GBM invariably recurs in patients regardless of age. A meta-analysis of Phase II trials reported that the proportion of patients without progressive disease (PD) at 6 months was only 15\% [11]. A multicenter retrospective analysis showed that elderly patients received below-standard care at the time of recurrence. Unlike a Karnofsky Performance Status (KPS) $<70$, age did not affect overall survival (OS) in this setting. The recommended treatment options at recurrence include repeat surgery, second-line chemotherapy and antiangiogenic agents [12].

To date, there is no defined standard treatment at recurrence. A safe, effective and acceptable therapy for elderly GBM patients is an important and unmet need [13,14].

Fotemustine (FTM) diethyl(1-[3-(2-chloroethyl)-3-nitrosoureido]ethyl)phosphonate is an alkylating cytotoxic agent belonging to the group of nitrosoureas, which have shown efficacy against gliomas. FTM enhances the

Future Medicine 
lipophilic properties, and its low molecular weight facilitates its passage through the blood-brain barrier and allows for significant diffusion in neuronal cells and glia [15-17].

According to the results of several studies, FTM represents a treatment option for recurrent GBM [18-25]. Despite its efficacy, the original FTM administration schedule, as first proposed by Khayat, is cumbersome and limited by the associated hematological toxicity. Notably, toxicity to the liver and kidneys is moderate [23,24].

In Addeo's schedule, FTM is administered in the induction phase at $80 \mathrm{mg} / \mathrm{m}^{2}$ on days $1,15,30,45$ and 60 followed by a 4-week rest period. Subsequently, maintenance therapy with FTM is administered at $80 \mathrm{mg} / \mathrm{m}^{2}$ every 4 weeks. The objective of this study was to assess the efficacy and safety of Addeo's alternative FTM administration schedule in elderly patients with GBM.

\section{Patients \& methods}

Inclusion criteria

Patients aged $>65$ years with histologically proven GBM, who underwent radiation therapy (RT) plus concomitant and adjuvant TMZ [26], and experienced subsequent progression/recurrence of disease were included in the study.

Progression was documented through MRI or computed tomography (CT) $\geq 3$ months after the end of RT or by evidence of PD on two consecutive radiologic examinations. For patients who were unable to undergo MRI (e.g., claustrophobia, ferromagnetic implant, foreign bodies, or implanted magnetic field-sensitive device), CT examination for all assessments was allowed. In any case, either MRI or CT evaluations were performed every 3 months for all the follow-up observation time. Patients were required to have a KPS $\geq 70$ at the initiation of chemotherapy with FTM, a minimum life expectancy of 3 months, measurable disease with contrast enhancement using MRI and/or CT (assessed within 2 weeks before study entry), and at least one lesion ( $2 \mathrm{~cm}$ in diameter) measurable by MRI or CT.

Other eligibility criteria included adequate hematologic function (white cell count $>2 \times 10^{9} / \mathrm{l}$, platelet count $>100,000 / \mathrm{mm}^{2}$ and hemoglobin $>8 \mathrm{~g} / \mathrm{dl}$ ), renal function (creatinine level $<2 \mathrm{mg} / \mathrm{dl}$ ) and liver function (AAT level $<1.5$-fold the upper limit of normal). The Institutional Ethical Committee of Frattamaggiore Sam Giovanni di Dio Hospital approved the protocol, and all patients provided informed consent before the initiation of treatment.

\section{Treatment plan}

FTM was administered according to Addeo's schedule [27]. In the induction phase, an $80 \mathrm{mg} / \mathrm{m}^{2}$ dose of FTM was administered over a $1 \mathrm{~h}$ infusion on days $1,15,30,45$ and 60 , followed by a 4 -week rest period. After this period, maintenance therapy was administered in nonprogressive patients at the same dose every 4 weeks until $\mathrm{PD}$, occurrence of unacceptable toxicity or patient withdrawal. Granulocyte-colony stimulating factor analogs were administered in patients with grade $\geq 1$ leukopenia or neutropenia according to the National Cancer Institute Common Toxicity Criteria, 3.0 version (2006).

In the case of toxicity occurrence, if the treatment was discontinued for $\geq 2$ weeks beyond the next scheduled cycle, the patient was permanently withdrawn from the study.

\section{Assessment}

For tumor evaluation, MRI or CT was performed at baseline, after the induction phase, before the maintenance phase and every three cycles thereafter. Imaging was also performed every time a progression of disease was clinically suspected. The MacDonald criteria [28] were uniformly adopted for the evaluation of response to treatment as follows:

- Complete response (CR): the disappearance of all lesions at two consecutive contrast-enhanced CT or MRI examinations performed $\geq 1$ month apart; neurologically stable or improved; and discontinuation of steroids;

- Partial response (PR): $\geq 50 \%$ reduction in the size of lesions at two consecutive contrast-enhanced CT or MRI examination performed $\geq 1$ month apart; neurologically stable or improved; and reduced or stable administration of steroids;

- PD: the presence of a new tumor or $>25 \%$ increase in the size of the original tumor at two consecutive contrast-enhanced CT or MRI examinations; stable or increased administration of steroids; or neurological worsening;

- Stable disease (SD): all other situations. 
The overall response rate (ORR) was defined as the proportion of CRs and PRs observed among all evaluable patients. The disease control rate (DCR) was defined as the proportion of CRs, PRs and SDs observed among all evaluable patients. The time to progression (TTP) was evaluated until PD was recorded from the end of RT in the Stupp protocol [26].

A neurological assessment was performed considering the results of previous examinations, as well as signs and symptoms according to local standard clinical practice. Of note, variations in the daily dosage of corticosteroids were recorded.

The extent of resection was assessed according to postoperative imaging and the operatory report.

Toxicity assessments were performed through clinical evaluation, and blood tests were performed every 2 weeks before the administration of each cycle of treatment during the induction phase and every 4 weeks during the maintenance phase, or more frequently if clinically indicated. Blood tests were performed weekly in case hematological toxicity necessitated a delay in the administration of chemotherapy.

The primary end point used to assess the efficacy of FTM was the proportion of patients free from PD or death due to any cause 6 months after the initiation of FTM (progression-free survival [PFS] rate at 6 months [PFS-6]). The loss of patients to follow-up before the 6-month time point was considered an event. Secondary end points were: OS measured from the initiation of FTM to death due to any cause or last follow-up assessment; ORR and DCR; TTP and toxicity (rate of events, mean number referred to any-grade toxicity, mean number referred to grade 3-4 toxicity).

Clinical characteristics, such as KPS, the presence of major comorbidity or obesity, and MGMT promoter methylation were recorded. Survival data (median survival times with $95 \%$ CIs) were computed through the Kaplan-Meier method and analyzed using the log-rank test and forward stepwise multivariate Cox proportional hazards model. The hazard ratios, together with their 95\% CIs, were computed. The statistical analyses were performed using the SPSS software (version 17.0 for Windows; SPSS, Inc., IL, USA). Two-tailed p-values $<0.05$ were considered statistically significant.

\section{Results}

\section{Patients}

From January 2012 to December 2014, 58 patients were enrolled at the following two centers: Oncology Department, 'S. Giovanni di Dio' Hospital in Frattamaggiore (Naples, Italy) and NeuroOncology Department Fondazione IRCCS 'Instituto Neurologico Carlo Besta' (Milan, Italy). The characteristics of the patients are summarized in Table 1. The median age was 68 years (range: $68-73$ years) and the median KPS was 80 . After revision of the files, the KPS scores of two patients were recalculated to be 50 (range: 50-100). These patients were included in the safety and efficacy analyses.

18 patients $(31 \%)$ had a $\mathrm{BMI} \geq 25$, while 23 patients (40\%) exhibited comorbidities at the time of enrollment: arterial hypertension, diabetes mellitus, hepatitis infection, chronic obstructive pulmonary disease or chronic heart failure.

All 58 patients were diagnosed with histologically confirmed GBM, and 47 patients (82\%) underwent macroscopic radical surgery. The MGMT promoter was methylated in 18 patients (36\%), whereas it was not methylated in 28 patients (56\%). The MGMT status was either not available or not evaluated in eight patients (14\%). All patients received RT with concurrent and adjuvant TMZ [20], regardless of the MGMT promoter methylation status. The median number of adjuvant TMZ cycles was six (range: 1-37 cycles). Overall, TMZ was well tolerated, despite the fact that almost all adverse effects were recorded in patients who received $>20$ cycles of treatment.

The median time to recurrence from RT was 10 months (range: 4-55 months). Of note, 30 patients $(52 \%)$ exhibited a time between RT and progression/recurrence $>12$ months. Twenty-three patients (40\%) underwent a second surgery after recurrence.

\section{Efficacy \& survival}

All patients were assessable for their response to treatment. The primary end point of the study, PFS-6, was $47 \%$ (27 patients) (Table 2). Overall, the median PFS was 6 months and the median survival was 7 months (Figure 1). Three CR (5\%), 14 PR (24\%) and 26 SD (45\%) were observed; the ORR was 29\% and the DCR was 74\%. Fifteen patients (26\%) experienced PD. The median PFS in responders versus nonresponders was 7 versus 3 months, respectively $(\mathrm{p}>0.0001)$. Moreover, the median OS was longer in responders versus nonresponders ( 8 vs 4 months, respectively; $\mathrm{p}=0.0002$ ). 
Table 1. Patient characteristics.

\begin{tabular}{|c|c|c|}
\hline Parameters & Number & Percentage (\%) \\
\hline Patients & 58 & 100 \\
\hline Gender: male & 37 & 64 \\
\hline Gender: female & 21 & 36 \\
\hline Age (years) & Median: 68 & Range: $65-73$ \\
\hline KPS $>90$ & 28 & 48 \\
\hline KPS $<90$ & 30 & 52 \\
\hline Initial grade: GBM & 58 & 100 \\
\hline MGMT: methylated & 18 & 36 \\
\hline MGMT: unmethylated & 32 & 64 \\
\hline MGMT: missing & 8 & 14 \\
\hline Obesity & 18 & 31 \\
\hline Comorbidities & 23 & 40 \\
\hline Stupp protocol & 58 & 100 \\
\hline Surgery & 47 & 81 \\
\hline Biopsy & 11 & 19 \\
\hline Second surgery & 23 & 40 \\
\hline TTP from RT: $<12$ months & 28 & 48 \\
\hline TTP from RT: $>12$ months & 30 & 52 \\
\hline TMZ cycles: $\mathrm{n}<6$ & 19 & 33 \\
\hline TMZ cycles: $n \geq 6$ & 39 & 67 \\
\hline
\end{tabular}

\section{Table 2. Activity end points.}

\begin{tabular}{lll} 
Parameter & Value & Confidence Interval \\
\hline Overall survival, median & 7 months & $95 \% \mathrm{Cl}$ \\
\hline Progression-free survival, median & 6 months & $95 \% \mathrm{Cl}$ \\
\hline Progression-free survival at 6 months & 27 months & $47 \% \mathrm{Cl}$ \\
\hline Disease control rate & 43 months & $74 \% \mathrm{Cl}$ \\
\hline
\end{tabular}

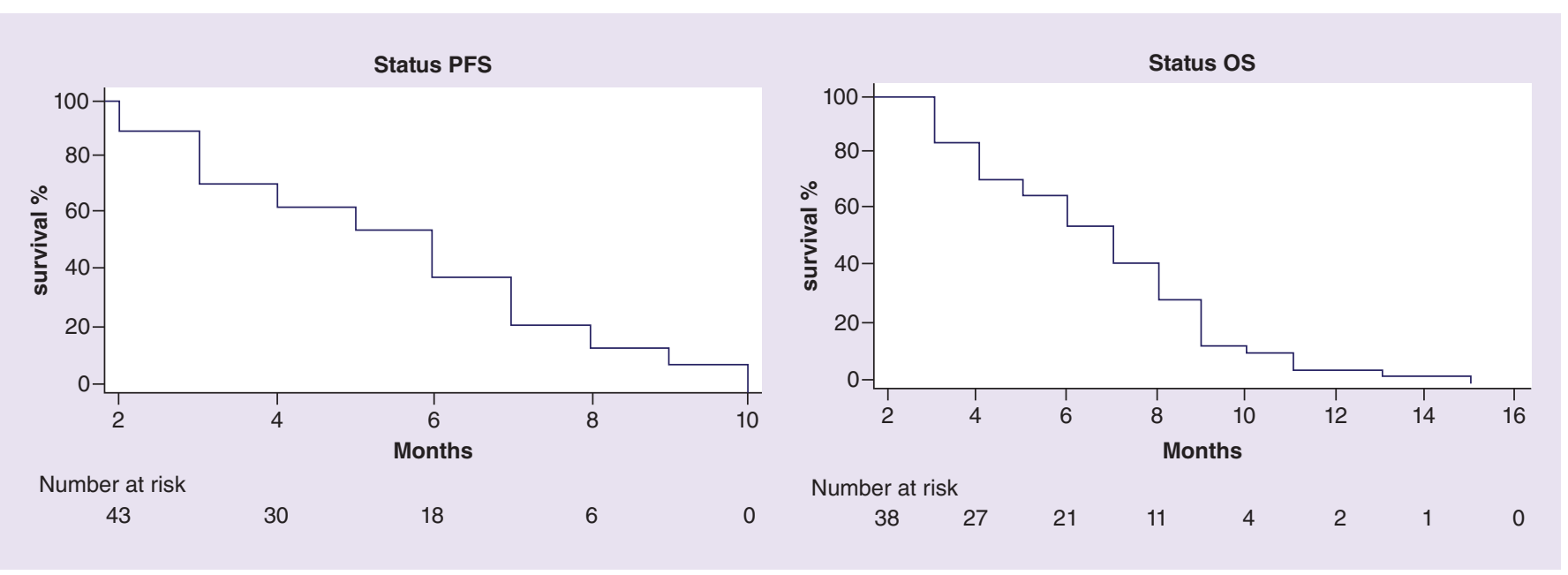

Figure 1. Progression-free survival (left) and overall survival (right) curves.Number at risk: Number of patients at the different observation times.

OS: Overall survival; PFS: Progression-free survival. 
Table 3. Incidence and grade of adverse effects (CTCAE 3.0).

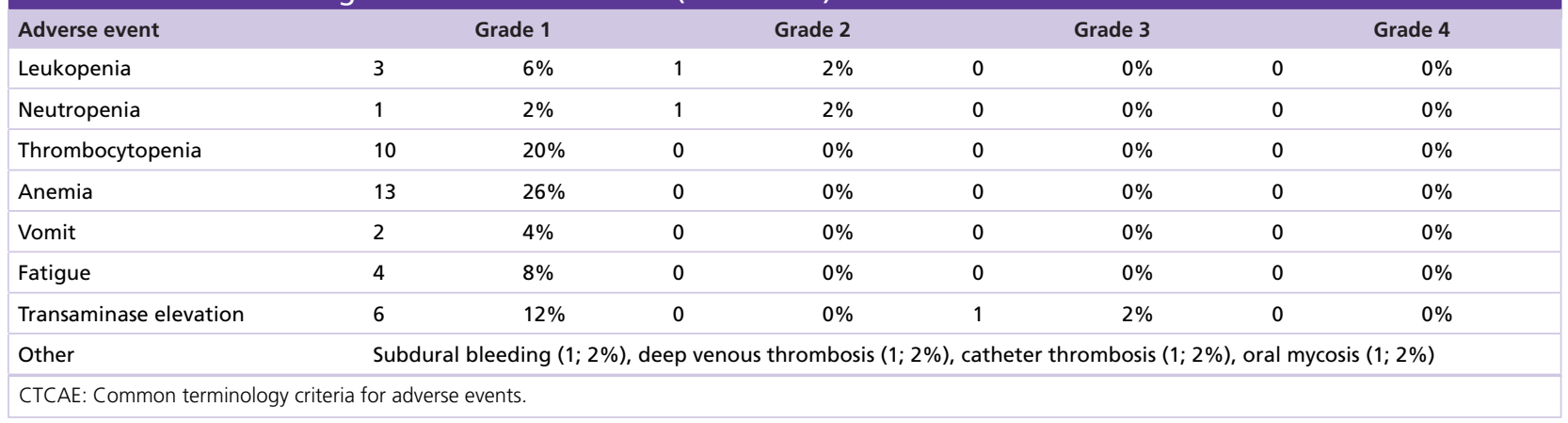

The multivariate analysis showed that a second surgery positively affected both PFS (7 vs 5 months, respectively; $\mathrm{p}=0.0069)$ and OS (9 vs 6 months, respectively; $\mathrm{p}=0.0301)$. Objective response, according to the McDonald criteria, played a similar role in both PFS (7 vs 3 months, respectively; $\mathrm{p}<0.0001)$ and OS $(8$ vs 4 months, respectively; $\mathrm{p}=0.0002)$. Longer TTP was positively related to longer PFS (6.5 vs 5 months, respectively; $\mathrm{p}=0.0085)$, but not to OS. In addition, the multivariate analysis revealed that PS ( $\mathrm{p}=0.41)$, comorbidity $(\mathrm{p}=0.71)$, obesity $(\mathrm{p}=0.13)$ and MGMT promoter methylation $(\mathrm{p}=0.30)$ did not affect PFS or OS.

\section{Toxicity}

All patients who received at least one dose of FTM were considered assessable for toxicity analysis. Fifty-three patients (91\%) completed the induction phase. Of those, 43 patients received at least one dose during the maintenance phase (overall rate: 74\%; rate accomplished over the induction phase: $81 \%$ ). Overall, toxicity (any grade) was observed in 22 patients (38\%; Table 3). There was no occurrence of grade 3-4 thrombocytopenia or neutropenia. Moreover, a low incidence of grade 3 elevation of transaminase $(2 \%)$ was reported with this schedule. The most commonly observed toxicity was grade 1 anemia (26\%).

In total, 477 cycles of FTM were administered. The median number of administered cycles was eight. Patients received a total of 194 cycles of FTM during the maintenance phase (the median number in this phase was three). The mean number of cycles required for the occurrence of toxicity (any grade) was 9.4, while that required for the occurrence of grade 3-4 toxicity was 412 .

\section{Discussion}

The results of the present study indicated that the biweekly administration of FTM was effective and safe in elderly patients with recurrent GBM. In addition, the identified positive prognostic factors were second surgery and objective response.

In recent years, the care for elderly patients with GBM has significantly improved [29]. First, standard RT improved survival in newly diagnosed GBM versus best supportive care alone [29,30]. Treatments with hypofractionated brain RT alone or TMZ are considered viable first-line options for GBM patients, even in elderly patients with KPS $\leq 60$. In fact, previous studies showed that hypofractionated RT (i.e., 40 Gy in 15 fractions) was noninferior to standard RT [29,31], and that TMZ alone and hypofractionated RT alone were superior to standard RT in terms of OS [11].

Currently, there is a lack of standard treatment strategies for elderly GBM patients. Several studies have demonstrated the efficacy of FTM against newly diagnosed or recurrent high-grade gliomas [32-35]. Previous trials investigating FTM reported an OS of 9-20 months, PFS of 4-9.1 months, PFS-6 of 27-48.5\% and DCR of 47.5-62\% [18-25].

According to the present results, the efficacy of Addeo's schedule in elderly patients with GBM is comparable to that observed in patients regardless of age [27]. In fact, in the original Phase II validation study of Addeo's schedule, the mean age of patients was 52.8 years (range: $30-75$ years), and the results were comparable. A similar biweekly FTM administration schedule was used in a Phase I/II trial involving patients aged 22-80 years [22]. The trial confirmed that biweekly administration of FTM was effective in inducing an ORR of $29 \%$ and a PFS- 6 of $47 \%$, without concerns regarding the occurrence of new toxicity.

An Italian series included 65 elderly patients (aged $\geq 65$ years; median age: 70 years) with recurrent GBM who were treated with FTM using the standard schedule [24]. Considering the limits of indirect comparison, the 
standard FTM schedule applied in this series performed worse versus the alternative schedule presented in this study. Specifically, in the previous series versus our study, the PFS, PFS- 6 and DCR were 4.2 versus 6 months, 35.4 versus $47 \%$, and 43 versus $74 \%$, respectively. Of note, the reported OS was similar in both studies (7.1 vs 7 months, respectively).

A standard definition of 'elderly' in the neuro-oncology community has not been established. However, approximately $50 \%$ of patients with GBM are aged $\geq 65$ years at the time of diagnosis [2]. Numerous studies, including the present study, define elderly patients using a cutoff age of 65 years $[4,24,36,37]$. However, other studies use a higher (i.e., 70 years) [29] or lower (i.e., 60 years) cutoff age [30]. The guidelines established by the National Comprehensive Cancer Network recommend a cutoff age of 70 years; however, patients are generally selected according to their KPS (i.e., KPS $<60$ vs KPS >60) [38]. Interestingly, in our study, KPS did not affect outcome. Moreover, it was not determined in the study performed by Santoni et al. [24]. However, in both studies, the response to treatment with FTM affected survival.

In our study, patients who underwent a second surgery were associated with both significantly improved PFS versus those who did not (7 vs 5 months, respectively; $\mathrm{p}=0.0069)$ and OS (9 vs 6 months, respectively; $\mathrm{p}=0.0301$ ). These finding were consistent with those reported in previous studies [39-42]. However, the low number of events $(n=15)$ limited our analysis of prognostic factors; this should be taken into consideration when interpreting these results.

In this study, Addeo's schedule was shown to be safe in GBM elderly patients, with a low incidence of adverse events versus that previously reported in the elderly population [27]. Gaviani and the Spanish GEINOFOTE Group also demonstrated the safety of this schedule in high-grade gliomas [34,43].

The interpreters of the results derived from the present study should take into account the good overall prognostic characteristics of our population. In fact, all patients completed the Stupp protocol as first-line treatment, and a high proportion (40\%) was able to undergo second surgery at the time of recurrence. Although this suggests a favorably selected population, the characteristics of patients included in this study are consistent with those of elderly GBM patients who would be offered second-line treatment in clinical practice.

\section{Conclusion}

Our study suggested that the biweekly FTM administration schedule might be safe and effective in elderly patients with recurrent GBM. This alternative schedule may be considered if further treatment is deemed feasible following a comprehensive assessment. Future studies should specifically investigate this population of GBM patients, and assess the benefits of different treatments in different subgroups according to tumor biomarkers. This approach may be useful in providing guidance for the selection of the most appropriate treatment [44-47].

\section{Future perspective}

The specific treatment of GBM is a challenging priority, and further research should be performed to identify alternative treatment modalities. For this subset of patients, the study of molecular alterations and the genetic fingerprint of GBM in the future may offer the opportunity to develop selective, self-tailored and mutationdependent therapeutic approaches [48-51]. Other specific contributions to the evolution of treatment against GBM may be derived from the delivery of anticancer drugs through the blood-brain barrier, and in tumor tissues through the use of nanocarriers [52-54]. The combination of these two approaches may generate an increase in the therapeutic index of anti-GBM agents, avoiding the occurrence of adverse effects, and allowing the effective treatment of elderly patients with recurrent GBM.

\section{Financial \& competing interests disclosure}

The authors have no relevant affiliations or financial involvement with any organization or entity with a financial interest in or financial conflict with the subject matter or materials discussed in the manuscript. This includes employment, consultancies, honoraria, stock ownership or options, expert testimony, grants or patents received or pending, or royalties.

Medical writing support was provided by Enago (www.enago.com) and was funded by personal private payment by the authors.

Ethical conduct of research

The authors state that they have obtained appropriate institutional review board approval or have followed the principles outlined in the Declaration of Helsinki for all human or animal experimental investigations. In addition, for investigations involving human 
subjects, informed consent has been obtained from the participants involved. The Institutional Ethical Committee of Frattamaggiore Sam Giovanni di Dio Hospital approved the protocol, and all patients provided informed consent before the initiation of treatment.

\section{Open access}

This work is licensed under the Attribution-NonCommercial-NoDerivatives 4.0 Unported License. To view a copy of this license, visit http://creativecommons.org/licenses/by-nc-nd/4.0/

\section{Summary points}

- Glioblastoma in the elderly is linked to a worse prognosis.

- The incidence of glioblastoma increases with age.

- In this study, patients aged $>65$ years were considered elderly, although there is no defined cutoff age for 'elderly' in the field of neuro-oncology.

- The alternative fotemustine administration schedule was effective in this population.

- The rate of progression-free survival at 6 months was $47 \%(n=27)$.

- The rate of objective response was $29 \%$, while disease control was reached in $74 \%$ of the patients.

- Median progression-free survival was 6 months, and longer in responders versus nonresponders (7 vs 3 months, respectively, $\mathrm{p}<0.0001$ ).

- Median survival was 7 months, and longer in responders versus nonresponders (8 vs 4 months, respectively, $\mathrm{p}=0.0002$ ).

- The incidence of toxicity (any grade) was 38\%; however, there was no grade 3-4 hematological toxicity (thrombocytopenia or neutropenia) reported.

\section{References}

Papers of special note have been highlighted as: $\bullet$ of interest; $\bullet \bullet$ of considerable interest

1. Ostrom QT, Gittleman H, Truitt G, Boscia A, Kruchko C, Barnholtz-Sloan JS. CBTRUS statistical report: primary brain and other central nervous system tumors diagnosed in the United States in 2011-2015. Neuro. Oncol. 20(4), iv1-iv86 (2018).

- Overviews the epidemiology of CNS tumors.

2. Snyder J, Walbert T. Managing glioblastoma in the elderly patient: new opportunities. Oncology (Williston Park) 31(6), 476-483 (2017).

- Describes the standard clinical management of glioblastoma (GBM) in elderly population.

3. Combs SE, Wagner J, Bischof $\mathrm{M}$ et al. Postoperative treatment of primary glioblastoma multiforme with radiation and concomitant temozolomide in elderly patients. Int. J. Radiat. Oncol. Biol. Phys. 70(4), 987-992 (2008).

- Describes the results derived from the concomitant radio- and chemotherapy in GBM in elderly population.

4. Fiorica F, Berretta M, Colosimo C et al. Glioblastoma in elderly patients: safety and efficacy of adjuvant radiotherapy with concomitant temozolomide. Arch. Gerontol. Geriatr. 51(1), 31-35 (2010).

- Describes the standard clinical management of GBM in elderly population.

5. Lee J-H, Jung T-Y, Jung $S$ et al. Performance status during and after radiotherapy plus concomitant and adjuvant temozolomide in elderly patients with glioblastoma multiforme. J. Clin. Neurosci. 20(4), 503-508 (2013).

6. Brandes AA, Franceschi E, Tosoni A et al. Temozolomide concomitant and adjuvant to radiotherapy in elderly patients with glioblastoma. Cancer 115(15), 3512-3518 (2009).

7. Okada M, Miyake K, Tamiya T. Glioblastoma treatment in the elderly. Neurol. Med. Chir. (Tokyo) 57(12), 667-676 (2017).

8. Morgan ER, Norman A, Laing K, Seal MD. Treatment and outcomes for glioblastoma in elderly compared with non-elderly patients: a population-based study. Curr. Oncol. 24(2), e92-e98 (2017).

- Describes the different treatment options and clinical outcomes in elderly and GBM populations.

9. Perry JR, Laperriere N, O'Callaghan CJ et al. Short-course radiation plus temozolomide in elderly patients with glioblastoma. N. Engl. J. Med. 376(11), 1027-1037 (2017).

- Describes the results derived from the combination between chemotherapy and radiotherapy in elderly GBM population.

10. Malmström A, Grønberg BH, Marosi C et al. Temozolomide versus standard 6-week radiotherapy versus hypofractionated radiotherapy in patients older than 60 years with glioblastoma: the Nordic randomised, Phase III trial. Lancet. Oncol. 13(9), 916-926 (2012).

- The first trial comparing temozolomide versus conventional radiotherapy versus hypofractionated radiotherapy in elderly GBM population.

11. Wong ET, Hess KR, Gleason MJ et al. Outcomes and prognostic factors in recurrent glioma patients enrolled onto Phase II clinical trials. J. Clin. Oncol. 17(8), 2572-2572 (1999). 
12. Zanello M, Roux A, Ursu R et al. Recurrent glioblastomas in the elderly after maximal first-line treatment: does preserved overall condition warrant a maximal second-line treatment? J. Neurooncol. 135(2), 285-297 (2017).

13. Young JS, Chmura SJ, Wainwright DA, Yamini B, Peters KB, Lukas RV. Management of glioblastoma in elderly patients. J. Neurol. Sci. 380, 250-255 (2017).

- Describes the optimal management of elderly GBM population.

14. Wick A, Kessler T, Elia AEH et al. Glioblastoma in elderly patients: solid conclusions built on shifting sand? Neuro. Oncol. 20(2), 174-183 (2018).

15. De Rossi A, Rossi L, Laudisi A et al. Focus on fotemustine. J. Exp. Clin. Cancer Res. 25(4), 461-468 (2006).

16. Addeo R, Zappavigna S, Luce A, Facchini S, Caraglia M. Chemotherapy in the management of brain metastases: the emerging role of fotemustine for patients with melanoma and NSCLC. Expert Opin. Drug Saf. 12(5), 729-740 (2013).

- Describes the activity of fotemustine (FTM) in the treatment of brain metastases from melanoma and lung cancer.

17. Brandes AA, Bartolotti M, Tosoni A, Franceschi E. Nitrosoureas in the management of malignant gliomas. Curr. Neurol. Neurosci. Rep. 16(2), 13 (2016).

18. Addeo R, De Santi MS, Del Prete S, Caraglia M. Fotemustine and recurrent glioblastoma: possible new opportunities for an old drug. Cancer Chemother. Pharmacol. 64(5), 863-866 (2009).

- Describes the activity of FTM in the second-line treatment of GBM patients.

19. Fabi A, Metro G, Russillo M et al. Treatment of recurrent malignant gliomas with fotemustine monotherapy: impact of dose and correlation with MGMT promoter methylation. BMC Cancer 9, 101 (2009).

20. Scoccianti S, Detti B, Sardaro A et al. Second-line chemotherapy with fotemustine in temozolomide-pretreated patients with relapsing glioblastoma: a single institution experience. Anticancer Drugs 19(6), 613-620 (2008).

21. Marinelli A, Lamberti G, Cerbone L et al. High-dose fotemustine in temozolomide-pretreated glioblastoma multiforme patients. Medicine (Baltimore) 97(27), e11254 (2018).

- Describes the activity of high-dose FTM in the second-line treatment of GBM.

22. Fabrini MG, Silvano G, Lolli I et al. A multi-institutional Phase II study on second-line fotemustine chemotherapy in recurrent glioblastoma. J. Neurooncol. 92(1), 79-86 (2009).

23. Khayat D, Giroux B, Berille J et al. Fotemustine in the treatment of brain primary tumors and metastases. Cancer Invest. 12(4), 414-420 (1994).

24. Santoni M, Scoccianti S, Lolli I et al. Efficacy and safety of second-line fotemustine in elderly patients with recurrent glioblastoma. J. Neurooncol. 113(3), 397-401 (2013).

25. Lombardi G, Bellu L, Pambuku A et al. Clinical outcome of an alternative fotemustine schedule in elderly patients with recurrent glioblastoma: a mono-institutional retrospective study. J. Neurooncol. 128(3), 481-486 (2016).

26. Stupp R, Mason WP, van den Bent MJ et al. Radiotherapy plus concomitant and adjuvant temozolomide for glioblastoma. $N$. Engl. J. Med. 352(10), 987-996 (2005).

- Describes for the first time the activity of concomitant radiotherapy plus temozolomide in the treatment of GBM.

27. Addeo R, Caraglia M, De Santi MS et al. A new schedule of fotemustine in temozolomide-pretreated patients with relapsing glioblastoma. J. Neurooncol. 102(3), 417-424 (2011).

- Describes a new low dose and continuous FTM schedule in the second-line treatment of GBM.

28. MacDonald D, Cascino T, Schold SJ, Cairncross J. Response criteria for Phase II studies of supratentorial malignant glioma. J. Clin. Oncol. 8, 1277-1280 (1990).

29. Minniti G, Lombardi G, Paolini S. Glioblastoma in elderly patients: current management and future perspectives. Cancers (Basel) 11(3), pii: E336 (2019).

30. Keime-Guibert F, Chinot O, Taillandier L et al. Radiotherapy for glioblastoma in the elderly. N. Engl. J. Med. 356(15), 1527-1535 (2007).

- Describes the activity of radiotherapy alone in the treatment of GBM in elderly population.

31. Matsuda K. Treatment outcomes of hypofractionated radiotherapy combined with temozolomide followed by bevacizumab salvage therapy in glioblastoma patients aged $>75$ years. Int. J. Clin. Oncol. 23(5), 820-825 (2018).

32. Prelaj A, Rebuzzi SE, Grassi M et al. Non-conventional fotemustine schedule as second-line treatment in recurrent malignant gliomas: survival across disease and treatment subgroup analysis and review of the literature. Mol. Clin. Oncol. 10, 58-66 (2019).

33. Beauchesne P. Fotemustine: a third-generation nitrosourea for the treatment of recurrent malignant gliomas. Cancers (Basel) 4(1), 77-87 (2012).

34. Pérez-Segura P, Manneh R, Ceballos I et al. GEINOFOTE: efficacy and safety of fotemustine in patients with high-grade recurrent gliomas and poor performance status. Clin. Transl. Oncol. 18(8), 805-812 (2016). 
35. Lombardi G, Farina P, Della Puppa A et al. An overview of fotemustine in high-grade gliomas: from single agent to association with bevacizumab. Biomed. Res. Int. 2014, 698542 (2014).

- Reviews the activity of FTM plus bevacizumab association in the treatment of GBM patients.

36. Roa W, Brasher PMA, Bauman G et al. Abbreviated course of radiation therapy in older patients with glioblastoma multiforme: a prospective randomized clinical trial. J. Clin. Oncol. 22(9), 1583-1588 (2004).

37. Zarnett OJ, Sahgal A, Gosio J et al. Treatment of elderly patients with glioblastoma: a systematic evidence-based analysis. JAMA Neurol. 72(5), 589-596 (2015).

- Summarizes the results derived from the evidence-based treatment of GBM in elderly population.

38. Burt Nabors L, Portnow J, Baehring J et al. NCCN guidelines version 1.2018 central nervous system cancers. 15(11), doi: 10.6004/jnccn.2017.0166 (2018).

39. Barker FG, Chang SM, Gutin PH et al. Survival and functional status after resection of recurrent glioblastoma multiforme. Neurosurgery 42(4), 709-720; discussion 720-3 (1998).

40. Brandes AA, Danieli D, Zunarelli E et al. Role of MGMT methylation status at time of diagnosis and recurrence for patients with glioblastoma: clinical implications. Oncologist 22(4), 432-437 (2017).

41. Dherijha M, Alakandy L, Barrett C. Treatment outcomes in newly diagnosed malignant glioma in elderly patients. Neuro Oncol. 20(1), i20 (2018).

42. Babu R, Komisarow JM, Agarwal VJ et al. Glioblastoma in the elderly: the effect of aggressive and modern therapies on survival. J. Neurosurg. 124(4), 998-1007 (2016).

43. Gaviani P, Simonetti G, Salmaggi A, Lamperti E, Silvani A. Safety of second-line chemotherapy with non-conventional fotemustine schedule in recurrent high grade gliomas: a single institution experience. J. Neurooncol. 113(3), 527-529 (2013).

44. Jordan JT, Gerstner ER, Batchelor TT, Cahill DP, Plotkin SR. Glioblastoma care in the elderly. Cancer 122(2), 189-197 (2016).

45. Chen W, Xu XK, Li JL et al. MALAT1 is a prognostic factor in glioblastoma multiforme and induces chemoresistance to temozolomide through suppressing miR-203 and promoting thymidylate synthase expression. Oncotarget 8(14), 22783-22799 (2017).

46. Mellai M, Cattaneo M, Storaci AM et al. SEL1L SNP rs12435998, a predictor of glioblastoma survival and response to radio-chemotherapy. Oncotarget 6(14), 12452-12467 (2015).

47. Schäfer A, Teufel J, Ringel F et al. Aldehyde dehydrogenase 1A1-a new mediator of resistance to temozolomide in glioblastoma. Neuro Oncol. 14(12), 1452-1464 (2012).

48. Ülgen E, Can Ö, Bilguvar $\mathrm{K}$ et al. Whole exome sequencing-based analysis to identify DNA damage repair deficiency as a major contributor to gliomagenesis in adult diffuse gliomas. J. Neurosurg. 5, 1-12 (2019).

49. Cantero D, Rodríguez de Lope Á, Moreno de la Presa R et al. Molecular study of long-term survivors of glioblastoma by gene-targeted next-generation sequencing. J. Neuropathol. Exp. Neurol. 77(8), 710-716 (2018).

50. Sandberg CJ, Altschuler G, Jeong J et al. Comparison of glioma stem cells to neural stem cells from the adult human brain identifies dysregulated Wnt- signaling and a fingerprint associated with clinical outcome. Exp. Cell Res. 319(14), 2230-2243 (2013).

51. Lino MM, Merlo A. PI3Kinase signaling in glioblastoma. J. Neurooncol. 103(3), 417-427 (2011).

52. Játiva P, Ceña V. Use of nanoparticles for glioblastoma treatment: a new approach. Nanomedicine 12(20), 2533-2554 (2017).

53. Salzano G, Zappavigna S, Luce A et al. Transferrin-targeted nanoparticles containing zoledronic acid as a potential tool to inhibit glioblastoma growth. J. Biomed. Nanotechnol. 12(4), 811-830 (2016).

- Describes the activity of new nanomedicine-based approaches in the treatment of GBM.

54. Porru M, Zappavigna S, Salzano G et al. Medical treatment of orthotopic glioblastoma with transferrin-conjugated nanoparticles encapsulating zoledronic acid. Oncotarget 5(21), 10446-10459 (2014).

- Describes the activity of new nanomedicine-based approaches in the treatment of GBM. 